\title{
Non-spatial and Spatial Linear Regression
}

\author{
Wouter Steenbeek, NSCR Stijn Ruiter, NSCR
}

This is an unedited draft, not for citation, of a book chapter that has been accepted for publication in E.Groff and C. Haberman (Eds.), The study of crime and place: A methods handbook (Temple University Press, 2021). Temple University Press retains all rights and subrights of this document. This pre-print is not published under a CC license.

Corresponding author: Wouter Steenbeek, Netherlands Institute for the Study of Crime and Law Enforcement (NSCR), The Netherlands. Email: wsteenbeek@nscr.nl 


\section{Introduction}

In "Crime and Place" research we are often interested in quantifying how crime relates to features of the environment. This requires the use of statistical models. In this chapter, we immediately dive into describing the workhorse of quantitative statistical analysis - linear regression analysis - and we describe some of its extensions we believe a student of "Crime and Place" should be familiar with. These models allow us to show how the phenomenon of interest (we use the term outcome variable denoted by $y$ in formulae) varies with something else (the predictor variable(s) denoted by $x$ in formulae). ${ }^{1}$

Throughout this chapter, we use data from the Washington, D.C., open data portal (https: //opendata.dc.gov/). Our outcome variable is the average annual number of robberies across the years 2015-2019, for which the portal provides detailed information. We used the open-source statistical programming environment R (R Core Team, 2020), and analytical steps are described step-by-step in the Supplementary material.

\section{Unit of analysis}

Before we can start analyzing the robbery data, we first need to decide what unit of analysis to use. In "Crime and Place" studies these typically are spatial units which range from addresses and street segments to census blocks and neighborhoods. This choice generally depends on the research question and it should be guided by theoretical considerations about the spatial scale at which the mechanisms we want to study operate. In spatial analysis, we always need to be aware that aggregation of spatial data into arbitrary areal units of different size and shape might give rise to the modifiable areal unit problem (MAUP) which could bias study results (Fotheringham \& Wong, 1991). So, deciding on a specific unit of analysis is something no analyst should do lightly or without giving alternatives some thought.

For this chapter we decided not to use administrative boundaries such as blocks or block groups, but to overlay the entire Washington, D.C. area with a regular grid of hexagons (see Supplementary material for additional information). In contrast to our statements above regarding MAUP, this was also an arbitrary choice not really governed by theoretical considerations about the spatial scale of the mechanisms at play, but rather made for didactical purposes. These 274 hexagons all have the same size (0.72 square kilometers) and the same number of neighboring units (apart from those at the boundaries), which simplifies nearest neighbor calculations compared to rectangular grid cells (Birch et al., 2007).

Having chosen hexagons as our spatial unit of analysis, we can plot the average annual robbery count in 2015-2019 per areal unit on the map of Washington, D.C. as shown in the left panel of Figure 1. It is immediately clear that robberies cluster in certain parts of the study area. A student of "Crime and Place" might have learned about some research that shows that robberies are more likely to occur at or close to places with liquor licensee locations. The right panel of Figure 1 shows the counts of all liquor licensee locations in Washington, D.C. per areal unit, which we obtained on September 19, 2020 from the Washington, D.C., open data portal. At first sight, it seems there is indeed some overlap between the two distributions displayed in Figure 1.

\footnotetext{
${ }^{1}$ The outcome variable is also known under many other names such as the dependent variable, response variable, explained variable, and regressand. A predictor variable is also known as independent variable, regressor, covariate, explanatory variable, or feature (in machine learning). Here we use predictor variable to emphasize that regression models predict an outcome; using the term explanatory variable hapharzardly might seem to suggest that we can always interpret the results of a statistical model causally, which is not the case. See, e.g., Hernán \& Robins (2020) for an introduction to causal inference.
} 
There are broadly speaking two types of questions the student could ask: questions related to causal inference and questions related to prediction (Stock \& Watson, 2020, p. 143). The following question is an example of the former: What would happen with the number of robberies if we were to reduce the number of liqour licensee locations? An example of the second type of question is: If we know some area has 100 liquor licensee locations, how many robberies do we expect in the area? The questions are similar in that they both pertain to how robberies and liquor licensee locations are related, but they are also different. For answering the first question, we need to estimate the causal effect of liquor licensee locations on robberies. For the second question, we need an estimate of the strength of the association, on average, between liquor licensee locations and robbery. How should we go about answering such questions? In this Chapter, we introduce some of the steps that you could take to answer the questions with a little more statistical rigor than by merely looking at how the two maps correspond.

\section{Robberies}

2015-2019

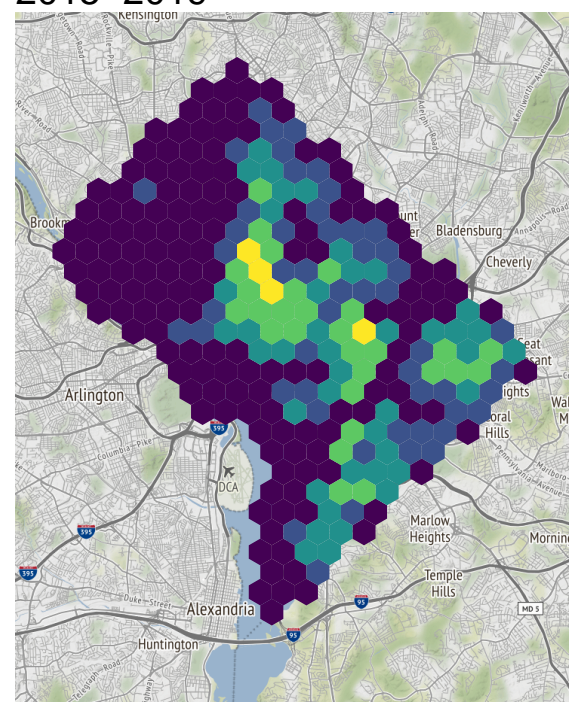

\section{Liquor licensee locations in 2020}

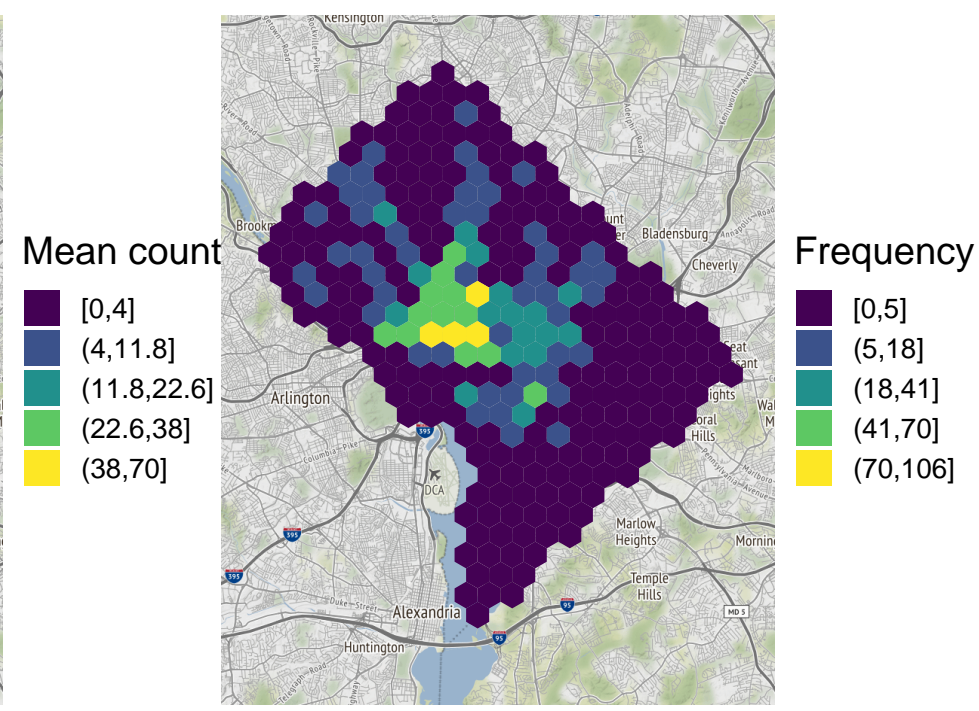

Figure 1: Robbery (left) and Liquor licensee locations (right) counts, mapped to hexagons

\section{Linear regression}

The average annual number of robberies per hexagon was 8.17 , but the robbery distribution is highly skewed. Some hexagons had no robberies in all five years, while the highest average annual number was 70. The median number of robberies was 3. Liquor licensee locations show a similar pattern: many hexagons did not have any liquor licensee location $(n=118)$, while hexagons have an average of 8 and a median of 1 liquor licensee locations.

The bivariate relation between the number of robberies and the number of liquor licensee locations in the hexagons is displayed in the left panel of Figure 2, in which each black circle represents one of the 274 hexagons. A visual inspection of this scatter plot again suggests that areas with more liquor licensee locations also have more robberies, as higher scores on the $x$-axis tend to 
correspond with higher values on the $y$-axis. It thus seems that there is an association between the two, but we still need to assess the strength of the association. Statistical models are the tools to do this.
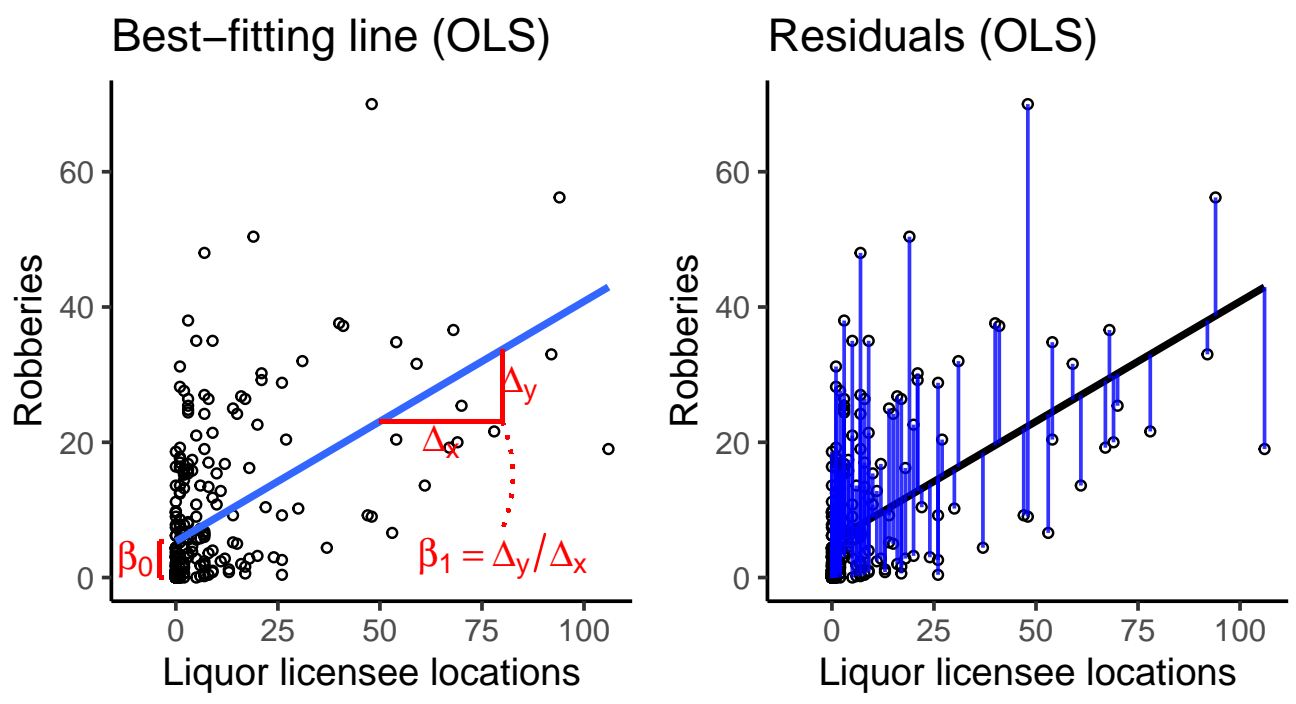

Figure 2: Bivariate relation between liquor licensee locations and robberies

\subsection{Statistical modeling: Generalizing from a sample to the population}

The purpose of a statistical model is to make inferences based on a limited set of data points (a sample) about some relation among a wider set of possible data points (the population). Although the assumptions are slightly different (see Stock \& Watson, 2020, Chapter 4.4 and Appendix 4.4), this applies to both causal inference as well as prediction. Drawing inferences based on a sample to conclude something about the population has everything to do with scientific discovery and the desire for generalizable knowledge. It starts from the idea that there should be some true model that captures the relation in the population. If we assume this relation between robberies $(y)$ and liquor licensee locations $(x)$ to be linear, the model could be written like this:

$$
y=\beta_{0}+\beta_{1} x+\epsilon,
$$

where both $\beta$-parameters describe how $y$ and $x$ are related, and the error term $\epsilon$ is included because we assume the relation to be probabilistic rather than deterministic, which means that some deviation from the line is to be expected.

\subsection{OLS regression}

The ordinary least squares (OLS) regression model is the simplest statistical model that provides esimates for the $\beta_{0}$ and $\beta_{1}$ parameters. Don't worry about the model name for now, it will become clear below. The OLS regression line is:

$$
\hat{y}=\hat{\beta_{0}}+\hat{\beta_{1}} x
$$

Note that $y$ of the true model is changed into $\hat{y}$ for the OLS regression line. This indicates that it is the expected (or predicted) $y$-value according to a statistical model based on a sample. The 
same holds for the $\hat{\beta}$-parameters: they are the model estimates for the unknown true population parameters. There is obviously no error-term $(\epsilon)$, because it is just a single regression line. The left panel of Figure 2 depicts the OLS regression line through all data points in blue. It runs from low values in the bottom-left to high values in the top-right, which is reflective of the positive relation between the two variables. As depicted (in red), $\beta_{0}$ indicates the height of the line at $x=0$, which is called the intercept of the regression line. $\beta_{1}$ denotes the slope of the regression line, which reflects how much the outcome variable $y$ changes with a unit increase in predictor variable $x$. When $\beta_{1}$ is positive, the line slopes upwards, as in our example. When $\beta_{1}$ is estimated to be negative, the line slopes downwards and there is a negative association between the predictor variable $x$ and the outcome variable $y$. If both variables are unrelated, $\beta_{1}$ would be zero, which results in a flat blue line because $y$ would not sytematically change across different values of $x$.

But how do we know that the blue line in the left panel of Figure 2 best reflects the association between robberies and liquor licensee locations? Why is it called the best-fitting line, and any other line with a different intercept and slope would fit the data worse? To understand this, we need to take a closer look at how OLS regression arrives at the estimated $\hat{\beta}$-parameters. The formula for the OLS regression model is:

$$
y_{i}=\hat{\beta}_{0}+\hat{\beta}_{1} x_{i}+\epsilon_{i} \quad(i=1, \ldots, n)
$$

Two things have changed compared to the OLS regression line above. First, $\epsilon$ has returned. Second, we now use subscript $i$ for $y, x$, and $\epsilon$ to indicate that it is a model for each individual data point $i$ $(i=1, \ldots, n)$ and not only for a single line through all points (one for each of the 274 observations in the data). $\epsilon_{i}$ is called the error term of the model. In the right panel of Figure 2, each blue line shows how much the data points differ from the best-fitting regression line. So each data point $i$ has an $\epsilon_{i}$. As can be seen in the figure, these are defined as:

$$
\epsilon_{i}=y_{i}-\hat{y}_{i}
$$

where $y_{i}$ refers to the observed values of the outcome variable (i.e. the robbery counts for each hexagon), and $\hat{y}_{i}$ denotes the predicted values according to the regression model (i.e. on the best-fitting line).

The estimated regression parameters or coefficients $\hat{\beta}_{0}$ and $\hat{\beta}_{1}$ are those that minimize the sum of the squared residuals, i.e. $\sum_{i=1}^{n}\left(y_{i}-\hat{y}_{i}\right)^{2}$. In other words, the parameters are estimated such that the line is as close as possible to all data points $i,(i=1, \ldots, n)$. Any other line with a different intercept and slope would lead to a larger sum of the squared errors, and would thus fit the data worse. It should now be clear why this particular regression model procedure is providing so-called least squares estimates. It was the first method developed around 1800 by Gauss and Legendre independently for obtaining estimates for the strength of an association. Since then, other and often more complex regression methods have been developed, which is why this first method is called the ordinary least squares (OLS) method. OLS regression is implemented in virtually all statistical software.

If we do not violate the OLS regression assumptions we discuss in more detail below, the parameter estimates we obtain with OLS regression are a good approximation of the true population parameters. In other words, by using OLS regression, we hope the following applies:

$$
\begin{aligned}
& \hat{\beta}_{0} \approx \beta_{0} \\
& \hat{\beta}_{1} \approx \beta_{1}
\end{aligned}
$$


In our example, OLS regression estimates the intercept $\hat{\beta}_{0}$ to be 5.34 and the slope $\hat{\beta}_{1}$ to be 0.35 . This means that according to this simple model, we would predict that hexagons without any liquor licensee locations would have an annual average of close to 5 robberies, while hexagons that have ten liquor licensee locations have (on average) about 3.55 more robberies per year (for a total of $5.34+3.55=8.89) .^{2}$ The regression line with the estimated parameters thus looks like this:

$$
\text { Robberies }=5.34+0.35 \times \text { Liquor licensee locations }
$$

\subsection{Standard errors of $\hat{\beta}$-parameters and confidence intervals}

The purpose of any statistical model, like the OLS regression model, is not only to estimate the key parameters that describe the relation between the variables of interest (i.e. the intercept and the slope of the best-fitting regression line in our example), but also to estimate the probability that these parameters are actually just the result of mere chance. Random sampling plays a key role here, because random samples have certain statistical properties that allow us to use statistics to calculate this probability.

Think of the parameters 5.34 and 0.35 as those that were estimated using only one specific sample. ${ }^{3}$ Imagine that we would now draw many new random samples and estimate the regression parameters over and over again. What parameter estimates would each sample yield? Definitely not exactly the same each time, but how much would they vary? If they would vary a lot, it means we should not be very confident that 5.34 and 0.35 are indeed close approximations of the true population parameters. If they would vary very little, we would be much more confident though. What we do know though, is that when we would resample randomly, the distribution of a parameter of interest would be approximately normal. This implies that the mean of this distribution would be equal to the population parameter.

Obviously, when we do research we generally cannot draw many more samples to repeat the estimation procedure. However, based on the central limit theorem, statisticians have developed methods that allow us to make inferences about the population parameters using only a single sample of data. For each regression parameter, OLS regression also estimates its standard error $(S E(\hat{\beta}))$. You could think of it as simply the standard deviation of the hypothetical distribution of parameter estimates that we would have obtained if we had resampled many times.

Table 1 shows how OLS regression results are often presented. The parameter estimates reflect how much $y$ changes with a unit change in $x$. Their respective standard errors are presented between parentheses.

Because the normal distribution is fundamental to the estimation procedure, Student $t$-tests can be used to assess whether the parameters are statistically significantly different from 0 . The $t$-values for each parameter can be calculated as $\hat{\beta} / S E(\hat{\beta})$. The $t$-value for the liquor licensee parameter is 10.32. The probability of observing such a $t$-value under the null hypothesis that the true effect is actually 0 turns out to be very small, namely $2.781 \mathrm{e}-21$. We conclude that it is highly unlikely to find these parameter estimates, and thus the null hypothesis is rejected. Often, effects are called statistically significant when the $p$-value is smaller than some predefined $\alpha$-level. In the social

\footnotetext{
${ }^{2}$ Researchers would often write that every additional liquor licensee location in a hexagon is expected to increase the average annual number of robberies by 0.35 , but this is very close to causal reasoning (e.g., compare this to "if we add another liquor licensee location to a hexagon, then we expect the average annual number of robberies to increase by $0.35 ")$, and we therefore caution the reader against using such language when causal claims are not warranted.

${ }^{3}$ Some readers might suddenly realize that we actually do not use a random sample, but in fact analyze all robberies that were officially registered in Washington, D.C., in the period 2015-2019. However, it is still recommended to use statistics and just treat the data as a sample out of a larger methaphorical population (Spiegelhalter, 2019) rather than assuming that our estimates would indeed be the true population parameters.
} 
Table 1: OLS regression results for robbery

\begin{tabular}{lc}
\hline \hline Liquor licensee locations & $0.35^{* * *}(0.03)$ \\
Intercept & $5.34^{* * *}(0.64)$ \\
\hline Observations & 274 \\
$\mathrm{R}^{2}$ & 0.28 \\
\hline \hline Note: & ${ }^{*} \mathrm{p}<0.05 ;{ }^{* *} \mathrm{p}<0.01 ;{ }^{* * *} \mathrm{p}<0.001$
\end{tabular}

sciences, $\alpha$-levels of 5,1 or even 0.1 percent are commonly used, and asterisks $(*)$ are displayed next to the statistically significant parameters. The corresponding critical absolute $t$-values for a two-sided significance test are 1.96, 2.58, and 3.3 respectively. Two-sided tests are used when we have not hypothesized the parameter to be in a certain direction. If, however, we would have hypothesized a positive relation between liquor licensee locations and robbery, we would not want to test whether the effect is statistically significantly different from 0 but actually that it is larger than 0 . In that case, we could have used a one-sided test for which the critical $t$-values are 1.65, 2.33 , and 3.1 respectively.

Standard errors can also be seen as measures of precision for our parameter estimates. If a standard error is relatively small compared to the parameter estimate, we can confidently conclude that the parameter is estimated with great precision. A large standard error thus reflects low precision. Another way to present this is by using confidence intervals, which are a translation of the standard error into the range within which the parameter estimate is likely to fall when it would have been estimated over and over again using new samples. The outer limits of the confidence interval are defined by the $\alpha$-level. That is, with an $\alpha$ of 5 percent, the estimated confidence interval ranges from $\hat{\beta}-1.96 \times S E(\hat{\beta})$ to $\hat{\beta}+1.96 \times S E(\hat{\beta})$ because 1.96 is the critical absolute $t$-value for a two-sided test. The confidence interval for the parameter estimate for liquor licensee locations thus ranges from 0.29 to 0.42 . In the remainder of this chapter, we will present confidence intervals in regression tables instead of standard errors, because this better communicates the expected range of the parameter estimate if we were to repeat the same study over and over again using a new sample every time.

\section{Key assumptions of OLS regression}

After obtaining the regression parameters, we could answer our predictive question. The predicted average annual number of robberies in a five year period in a hexagon area with 100 liquor licensee locations is $5.34+0.35 * 100=40.34$. However, knowing what to expect is not the same as knowing what would happen if we were to change the number of liquor licensee locations in an area. For that we need to know what the causal effect is of liquor licensee locations on robbery. Next to their predictive purpose, regression models are also used for causal inference. Causal inference is at the heart of science, as the end game of science is to know what causes what. Medical doctors want to know what causes and cures certain diseases, political scientists try to understand why people vote the way they do, and criminologists study why crime clusters among places, offenders, and victims.

We cannot simply assume the estimated parameter of liquor licensee locations to reflect the causal effect of a liquor licensee location on the average annual number of robberies. For example, perhaps there is a common cause of both the presence of liquor licensee locations as well as the occurrence of robberies. OLS regression models cannot prove causality, but they are often helpful or even necessary 
tools to start evaluating and testing causal claims. In most cases, they only summarize the statistical relation for predictive purposes, but under certain assumptions the parameter estimates reflect causal effects. Hernán \& Robins (2020) and Cunningham (2018) provide extensive explanations of (the difficulties of) establishing causality through non-experimental designs, but here we list the least squares assumptions for causal inference (Stock \& Watson, 2020, Chapter 4.4).

A violation of any of the assumptions potentially leads to biased estimates. Although OLS regression has proven to be quite robust against small violations, it is always important to carefully run model diagnostics before drawing any conclusions based on a statistical model (see the Supplementary material for a number of tools for model diagnostics). Perhaps the first thing we should do, however, is to emphasize plotting your data. We are estimating a regression model that assumes a linear relationship between $x$ and $y$ so it can never hurt to just create scatter plots. This may already lead you to new ideas and to question the linearity assumption, before you even dive into more formal diagnostic checks. ${ }^{4}$

Assumption 1: The conditional distribution of $\epsilon_{i}$ given $x_{i}$ has a mean of 0 . There might be multiple predictors related to $y$. If some of them were not included into the model, $\epsilon_{i}$ will represent the contribution to $y$ of these omitted variables. This assumption asserts that all omitted variables are uncorrelated with $x_{i}$. The assumption definitely holds when $x_{i}$ would have been randomly assigned, but in observational designs this is rarely the case and there are obviously no tests for whether $\epsilon_{i}$ is uncorrelated with any unknown variable not included in the model.

Assumption 2: $\left(x_{i}, y_{i}\right), i=1, \ldots, n$ are independently and identically distributed across observations.

This i.i.d. assumption basically holds under random sampling, as observations randomly sampled from a population will have the same distribution and random draws guarantee they are not related and thus independent. This assumption can be violated in several ways. For example, with time series or panel data, there is often serial autocorrelation. This means that the error term of an observation is correlated with the error term of the next observation. This can easily be checked by plotting $\epsilon_{i}$ in the order in which the data were collected. When dealing with spatial data, which is so fundamental to "Crime and Place" studies, $\epsilon_{i}$ could also be spatially autocorrelated (as discussed later in this chapter).

Assumption 3: Large outliers are unlikely.

This assumption means that observations with extreme (positive or negative) values on $x_{i}$ or $y_{i}$, far beyond the usual range of values, are unlikely. Influential observations are those that have a big impact on the parameters. These can be observations with extreme scores on $y$ or $x$, because especially those observations will have a large impact on how the regression parameters get estimated. In order to detect influential observations, first the outliers need to be detected. These are the observations with relatively large (so-called 'studentized') residuals. It should then be determined how much each individual observation affects the model parameters, by assessing whether the model parameters would change much by excluding observations from the model. That's not to say that such observations have to be removed from the model, but they definitely need to be scrutinized. For example, such extreme values are occasionally simply the result of data entry errors, which should then be corrected.

If these three assumptions hold, OLS estimators are consistent and their sampling distributions will be normal in large samples, and we can use the model results for causal inference. In practice, and

\footnotetext{
${ }^{4}$ Note: A linear regression model does not preclude the estimation of non-linear relationships, e.g., by including additional $x$-variables as polynomials or exponential functions.
} 
particularly with observational designs, it is however hard to justify whether they hold, especially assumptions 1 and 2. When OLS regression models are estimated using small samples, two additional assumptions are required in order to rely on the OLS test statistics for causal inference (see Stock \& Watson, 2020, sec. 5.6 and Chapter 18 for details).

Assumption 4: $\epsilon_{i}$ has a constant variance.

This is the homoskedasticity assumption, which states that the variance of the errors around the regression line is the same across the values of the predictor variables. If you erroneously assume homoskedasticity, the parameter estimates are unbiased (we can still trust the point estimate) but inefficient (the estimates of the standard errors are biased). We can check this assumption by plotting the residuals $\epsilon_{i}$ against the fitted values $\hat{y}_{i}$, or using the Breusch-Pagan test.

Assumption 5: the conditional distribution of $\epsilon_{i}$ given $x_{i}$ is normal.

The distribution of $\epsilon_{i}$ can be checked with, e.g., a residual Q-Q plot, plotting the histogram of residuals, and a scatter plot of the model's residuals and the fitted values.

\subsection{Multiple regression: OLS regression with multiple predictor variables}

So far, we have discussed the simplest OLS regression model with a single predictor variable (i.e. liquor licensee locations). This model can easily be extended to a model with more than one $(k)$ predictor variables. The rest remains virtually the same. The formula for this extended model is:

$$
y_{i}=\hat{\beta}_{0}+\hat{\beta}_{1} x_{1 i}+\hat{\beta}_{2} x_{2 i}+\cdots+\hat{\beta}_{k} x_{k i}+\epsilon_{i} \quad(i=1, \ldots, n)
$$

There are often very good reasons to include more than one predictor in a regression model. If our aim is prediction, including more predictor variables generally yields better predictions. When we want to test causal claims, among other things there should not be another explanation for why $x$ and $y$ are related. From the results presented earlier, we cannot simply conclude that liquor licensee locations cause robberies. What if liquor licensee locations are often near grocery stores or other facilities, and robbers are actually attracted to these other facilities? Because liquor licensee locations tend to cluster in the same areas as such other facilities (but the latter facilities are not included as variables in the model), we would erroneously conclude that liquor licensee locations cause robbery.

Generally speaking, if there are other predictor variables that are correlated with $x$ and a determinant of $y$, the parameter for $x$ will be biased when these other predictor variables are not included in the model. This is called omitted variable bias (see assumption 1). ${ }^{5}$ Should we have data on these omitted variables, it thus makes great sense to include them into the model. Only if we still find an effect of $x$ after we included all omitted variables, we can be more confident about a causal claim. It still is no proof, and there might still be other omitted variables for which we had no data, but at least we would have an estimate of the effect of $x$ while adjusting for all other variables that are correlated to $x$ and also affect $y$. At the same time, it is incorrect to assume that we should therefore saturate regression models with all possible predictor variables, because we can also introduce bias (and inefficiency) by including predictor variables. We refer the reader to, e.g., Hernán \& Robins (2020) and Steiner \& Kim (2016) for further discussion on this expansive topic.

Another reason not to include too many predictor variables, especially if they are highly correlated, is that multiple regression models could suffer from multicollinearity issues. Multicollinearity exists when one or more predictor variables are a (near) linear function of a combination of some of

\footnotetext{
${ }^{5}$ Note, however, that the mechanics of omitted variable bias are quite complex. For example, see Steiner \& Kim (2016).
} 
the other predictor variables. In case of multicollinearity, OLS regression cannot estimate how much each predictor variable uniquely contributes to $y$, because the variables simply overlap. Most statistical software implements procedures to assess whether multicollinearity is an issue.

\subsection{Multiple linear regression results}

Suppose now that grocery stores indeed attract robbers. Suppose also that liquor licensee locations are often found nearby grocery stores. It could then be that the parameter estimate we found for liquor licensee locations is for a large part - or even entirely - driven by the effect of grocery stores on robbery. The multiple regression model permits the estimation of an effect on $y$ of changing a single variable $x$ while adjusting for all other variables. So, by including multiple predictor variables, we try to isolate the unique contribution of each.

Table 2 presents the results of an OLS regression model for robbery that includes, next to liquor licensee locations, the number of grocery stores, schools (both as continuous variables), and whether there was an ATM present in the hexagon (as dichotomous variable). The first model presents unstandardized regression parameters. Again, we can interpret these as how much (on average) hexagons differ in $y$ when they differ by one unit in $x$. However, because the model now includes other predictors too, the estimate for liquor licensee locations (0.22) is conditional on the other predictors in the model. This is another way of saying that it is the estimated effect while holding all other variables constant. Notice that the estimate for liquor licensee locations is considerably smaller than what we found in Table 1.

Table 2: Multiple regression results for robbery

\begin{tabular}{lcc}
\hline \hline & Unstandardized & Standardized \\
Liquor licenses & $0.22^{* * *}(0.14,0.29)$ & $3.71^{* * *}(2.44,4.98)$ \\
Grocery stores & $4.14^{* * *}(2.08,6.20)$ & $2.26^{* * *}(1.13,3.38)$ \\
Schools & $5.46^{* * *}(4.24,6.69)$ & $4.46^{* * *}(3.46,5.46)$ \\
ATM & $1.30(-1.64,4.24)$ & $1.30(-1.64,4.24)$ \\
Intercept & $2.84^{* * *}(1.65,4.03)$ & $7.84^{* * *}(6.62,9.06)$ \\
\hline Observations & 274 & 274 \\
$\mathrm{R}^{2}$ & 0.49 & 0.49 \\
\hline \hline Note: & & ${ }^{*} \mathrm{p}<0.05 ;{ }^{* *} \mathrm{p}<0.01 ;{ }^{* * *} \mathrm{p}<0.001$
\end{tabular}

The second model in Table 2 presents the standardized regression parameters of exactly the same regression model. Standardization helps when we want to compare the size of multiple parameters (in this case there are four plus the intercept), which says something about the relative influence of different variables. Standardized parameters are obtained by estimating the model with standardized continuous variables instead of unstandardized $x$-variables (the dummy variable ATM remains unchanged). Continuous $x$-variables can be standardized by subtracting the mean from the value for each case, and then dividing this difference by its standard deviation. This way, the estimated parameter expresses the difference in $y$ given a one standard deviation difference in $x$. As can be seen in Table 2, hexagons that score one standard deviation higher on liquor licensee locations have about 3.71 more robberies. The standardized parameter estimate for schools is even larger (4.46) and that of grocery stores is smaller (2.26). Although the parameter estimate for ATM is positive and it thus suggests that the presence of an ATM corresponds with a higher number of robberies, the parameter estimate is not statistically significant. 
Figure 3 shows the standardized regression parameters with their corresponding confidence intervals graphically. Notice that three types of confidence intervals are presented. The estimated OLS standard error only applies when the regression assumptions hold. When however the residuals violate some of the distributional assumptions, the parameter estimate $\beta$ might still be unbiased, but it is better to use heteroskedasticity-consistent standard errors of which two are displayed (HC1 and HC3). These robust standard errors are generally larger and the confidence interval is thus wider than the OLS results, leading to more conservative hypothesis testing (i.e. we reject the null hypothesis less often). Better yet is to carefully go through all diagnostic steps in order to find why the model is misspecified and try to fix it.

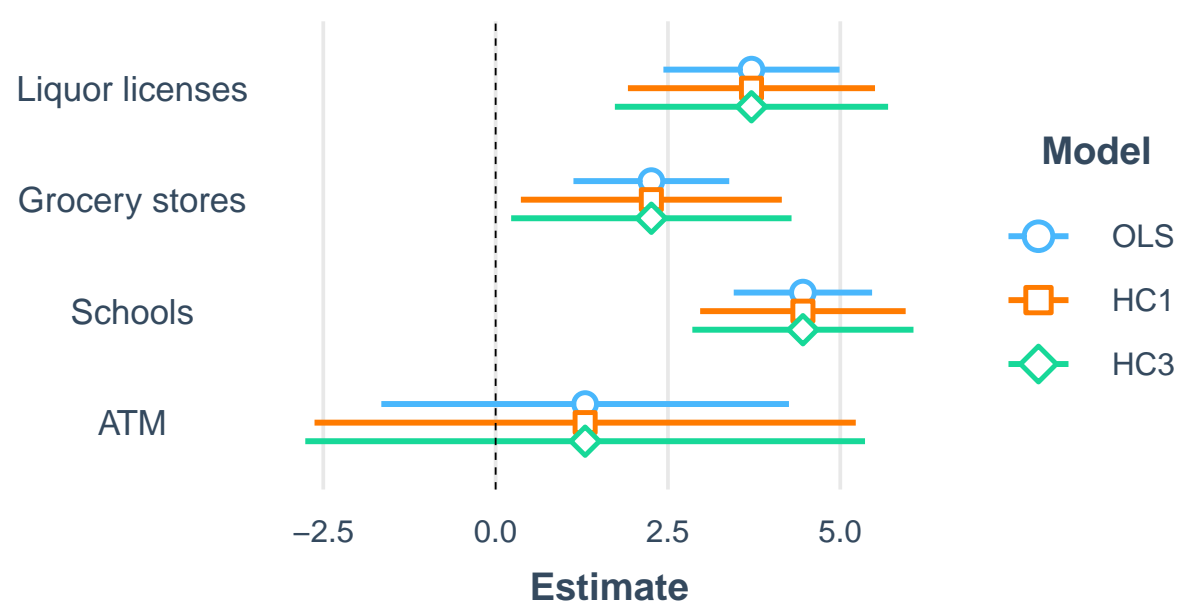

Figure 3: Standardized regression coefficients plot

Table 1 and 2 not only presented the parameter estimates and their respective standard errors or confidence intervals, but also the $R^{2}$ of the OLS regression models. It is a statistical measure for the proportion of the variance of the outcome variable that is explained by the predictor variables. Its value ranges from 0 to 1 , with low values indicating that the predictor variables do not do a good job predicting the observed outcome values. A value of 1 would mean that the set of predictor variables would perfectly predict the outcome values: all predicted values would be spot-on. As such, $R^{2}$ is inversely related to the residual error $\epsilon$ : a good fitting model (with small residual error) has a high $R^{2}$, and a model with large residuals has a low $R^{2}$.

The $R^{2}$ is often used to compare models. For example, the $R^{2}$ of the model presented in Table 1 is 0.28 . This means that $1-0.28=0.72$ of the variance in robbery remains unexplained. By including three additional variables in Table 2 , the $R^{2}$ increases to 0.49 , and the model thus better fits the data. Because the $R^{2}$ of a model always increases with the inclusion of additional predictor variables - even if the additional variables provide little to no improvement - it is actually ill-suited for choosing between different models. For model selection, other model fit statistics provide a better help. The adjusted- $R^{2}$ scales the $R^{2}$ downward with the inclusion of too many predictor variables in the model. The Bayesian Information Criterion (BIC) and the Akaike Information Criterion (AIC) also penalize the inclusion of additional variables to a model, and models with a lower $\mathrm{BIC}$ and $\mathrm{AIC}$ are considered better-fitting. 


\section{Linear spatial regression}

\subsection{Why might we need spatial models?}

The regression models discussed thus far assumed a data generating process for a sample of $n$ independent observations $y_{i}, i=1, \ldots, n$ that are linearly related to predictor variables. In "Crime and Place" studies, the units of analysis are often spatial units such as street segments, census blocks or hexagons, and the data are not a random sample but cover a complete study area (e.g., an entire city). So, when we analyze areal units like street segments or census blocks with the equation above, we effectively assume that the observed values at one location are independent of the observed values at other locations. How realistic is this assumption in a spatial context? Not very. Tobler's first law of geography states that "everything is related to everything else, but near things are more related than distant things" (Tobler, 1970). This simple statement is also at the heart of spatial criminology. Crime does not occur randomly across space: high-crime areas are often found near other high-crime areas, and low-crime areas near other low-crime areas (see also Chapter 'Spatial Autocorrelation' in this handbook). Of course, the question remains why this is the case. The challenge is to explain why crime clusters in some areas and not in others.

Tobler's law as well as overwhelming empirical evidence of spatial clustering of crime imply that locations indeed exhibit spatial dependence, and this violates assumptions of ordinary least squares regression analysis. Because of the clustering, each unit of analysis contributes less new information to the model than if observations would have been independent. To make valid inferences, we need to use regression models that can deal with the spatial dependence.

We also often have solid theoretical reasons why we expect that nearby locations are more alike than distant locations. There are three main reasons why observations of the outcome variable could be spatially dependent. Following our example of robbery in hexagons, this is first, due to exogenous interaction, which means that phenomena in nearby hexagons affect robbery in the focal hexagon. Second, due to endogenous interaction, whereby crime in the focal block depends on the crime level of nearby blocks. In this case, any spatial effects of the predictor variables are assumed to work indirectly through their influence on crime. Third, due to interaction among error terms. Here we do not require a theoretical model; it can occur for various reasons like when the spatial scale of the model (here, hexagons) does not match the spatial scale of the phenomenon (e.g., when it operates at the street segment level); measurement error in the observations; failure to include an important predictor variable (i.e. omitted variable bias), which had a spatially autocorrelated structure; and misspecified functional form of the regression parameters.

\subsection{Spatial neighbors and spatial weights}

The next sections depend on the specification of the spatial weights matrix $(W)$, which is why we need a small detour to explain what this refers to. The spatial weights matrix captures our ideas about the spatial dependency relation between our units of analysis: (1) which spatial units can affect which other spatial units (this is the choice of neighbors)?; (2) in what way do these spatial units then affect the focal spatial unit? Together, these decisions are reflected in the way a $W$ matrix is specified.

For example, we might assume only adjacent spatial units to affect the focal spatial unit (a.k.a. first-order spatial lags). Alternatively, maybe a focal unit is affected by its $k$ nearest neighboring units (where $k$ is a positive integer). Actually, we can specify any spatial structure we want by manipulating the $W$ matrix (although some conditions will need to be imposed to obtain consistent estimators of the parameters). Contextual knowledge and theoretical justification when specifying $W$ are key, and thus no standard guidelines can be given, which makes specifying the weights matrix 
a challenge. Please see the Supplementary material for more details, as well as Chapter 'Spatial Autocorrelation' in this handbook.

An important caveat is that in order to perform a correct test, e.g., of spatial autocorelation of the OLS residuals in the next section, the choice of spatial weights must match the true spatial process. If we use spatial weights that do not match the true spatial process - and especially if the spatial effect is not very strong - we will not reject the null-hypothesis of no spatial autocorrelation even though it is present. Therefore, the specification of the spatial weight matrix is a very important decision made by the analyst, and for this reason we discuss this in more detail in the Supplementary material. Unfortunately, no general advice can be given on the spatial weights specification, and contextual knowledge of the problem at hand is key (e.g., see Tita \& Radil, 2010).

\subsection{Spatial autocorrelation of OLS residuals}

The final model in the previous section used liquor licensee locations, grocery stores, schools, and the presence of an ATM to predict the average annual number of robberies in hexagons in Washington, D.C. If this model does not capture the true data generating process of Robbery because, for example, we have erroneously omitted the presence of nearby ATMs and liquor stores, the model's residuals $\left(\epsilon_{i}\right)$ may exhibit spatial autocorrelation. The concept of spatial autocorrelation, as well as a number of different statistics that provide information on the level of global and local spatial autocorrelation, was already discussed in Chapter 'Spatial Autocorrelation.' Those ideas are directly applicable here, but instead of the spatial autocorrelation of the outcome variable, we investigate the spatial autocorrelation of the residuals.

Spatial autocorrelation of the model's residuals can be assessed using Moran's $I$. Here, we assume a row-standardized "rook" 1st-order weight matrix. A two-sided test on the residuals returns a global Moran's $I$ of 0.37 for our OLS multiple regression model, which is statistically significant. This leads us to reject the null hypothesis of uncorrelated error terms. The statistic is positive, indicating that hexagons with positive OLS residuals - i.e. more robbery than expected - are typically surrounded by hexagons that also experience more-than-expected robbery. Global tests for spatial autocorrelation provide a summary statistic of the local relationships between focal spatial units and their neighboring spatial units. The global Moran's $I$ value can be decomposed in order to show for which units of analysis the OLS residuals and their spatially-lagged residuals correlate.

Now that we have found statistically significant spatial autocorrelation of the OLS residuals (see Supplementary material), we still should not jump to the conclusion that we need a more advanced spatial regression model. We might have forgotten to include an important predictor variable in the OLS model, which, if it had been included, would have captured the observed spatial autocorrelation in the OLS residuals. Spatially autocorrelated residuals could also indicate other types of model misspecification, such as non-linear effects of predictor variables. In our example, we fitted a linear OLS regression model to the count variable Robbery instead of a(n overdispersed) Poisson regression model, which could also be partly responsible for why we now detect spatial autocorrelation. Bivand et al. (2013, p. 289) emphasizes that in these cases one should always first try to improve the model itself (e.g., by including the missing predictors) rather than spend effort on modeling the spatial structure.

\subsection{An example: spatially-lagged predictor variable (SLX model)}

What if we do have reason to believe that we have a well-specified regression model and the spatially autocorrelated residuals are not due to other sources of model misspecification? Spatially autocorrelated residuals could be seen as a nuisance that should be solved by estimation methods 
that allow for spatial dependence of the regression residuals. However, we prefer to see spatial autocorrelation first and foremost as an interesting phenomenon that we ideally would like to model. Therefore, we first discuss the spatially-lagged $X$ (SLX) model using an example. In the next section, we discuss a number of spatial regression models that allow us to incorporate explicitly the three spatial interaction mechanisms in the regression equation.

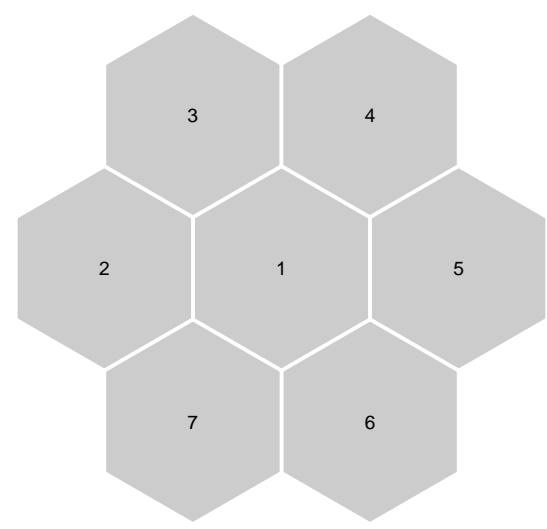

Figure 4: Seven hexagons

Let's focus on seven of the hexagons covering Washington D.C., as depicted in Figure 4. Suppose our hypothesis is that offenders rob victims after they visit ATMs, and thus our model should reflect that robbery (our outcome variable $y$ ) in the focal hexagon is affected by the presence of an ATM (predictor variable $x$ ) in the focal hexagon $i: y_{i}=\beta_{0}+\beta_{1} x_{1, i}+\epsilon_{i}$. This is the standard regression model, which assumes that regardless of location, the presence of an ATM $\left(x_{1}\right)$ has the same effect on robbery (captured by $\beta_{1}$ ). For each of the seven hexagons, we could also write out the equations so you can see the full data generating process (note that in the remainder we refer to the population regression parameters $\beta$ rather than the ordinary least squares estimators of these parameters, $\hat{\beta}$ ):

$$
\begin{aligned}
y_{1} & =\beta_{0}+\beta_{1} x_{1,1}+\epsilon_{1} \\
y_{2} & =\beta_{0}+\beta_{1} x_{1,2}+\epsilon_{2} \\
y_{.} & =\ldots \\
y_{7} & =\beta_{0}+\beta_{1} x_{1,7}+\epsilon_{7}
\end{aligned}
$$

where $x_{1, i}$ refers to the value of $x_{1}$ in hexagon $i$ and $i=1, \ldots, n$ hexagons. Simply put, this model assumes an overall intercept $\beta_{0}$ which captures the level of robbery when $x_{1}$ equals zero and one estimate of the effect of $x_{1}$ on robbery, $\beta_{1}$. To predict robbery in hexagon $i$, we multiply $\beta_{1}$ by the value of $x_{1}$ in hexagon $i\left(x_{1, i}\right)$.

In addition, however, we might also expect the ATMs nearby to matter: robbers may also follow victims for a short while before striking, perhaps because there are fewer witnesses around at some distance from the ATM. We thus expect the predictor variable to also assert an influence some distance away from its location. We might think of a very nonrestrictive model, where we assume that robbery in each hexagon depends on the presence of ATMs in all other hexagons, and furthermore that these spatial effects are unique from hexagon to hexagon. A model that captures that the value of $x_{1}$ in each hexagon $j$ can affect robbery in the focal hexagon $i\left(x_{1, j}\right)$ includes many coefficients of $\beta_{j, i}$, which refers to the effect of the predictor variable $x_{1}$ of hexagon $j$ on robbery in hexagon $i$. 


$$
\begin{aligned}
& y_{1}=\beta_{0}+\beta_{1} x_{1,1}+\left(\beta_{2,1} x_{1,2}+\beta_{3,1} x_{1,3}+\beta_{4,1} x_{1,4}+\beta_{5,1} x_{1,5}+\beta_{6,1} x_{1,6}+\beta_{7,1} x_{1,7}\right)+\epsilon_{1} \\
& y_{2}=\beta_{0}+\beta_{1} x_{1,2}+\left(\beta_{1,2} x_{1,2}+\beta_{3,2} x_{1,3}+\beta_{4,2} x_{1,4}+\beta_{5,2} x_{1,5}+\beta_{6,2} x_{1,6}+\beta_{7,2} x_{1,7}\right)+\epsilon_{2} \\
& y_{. .}=\ldots \\
& y_{7}=\beta_{0}+\beta_{1} x_{1,7}+\left(\beta_{1,7} x_{1,1}+\beta_{2,7} x_{1,2}+\beta_{3,7} x_{1,3}+\beta_{4,7} x_{1,4}+\beta_{5,7} x_{1,5}+\beta_{6,7} x_{1,6}\right)+\epsilon_{7}
\end{aligned}
$$

It is easy to recognize the standard regression model and the added spatial effects between parentheses. Ideally, we would like to know each and every one of these $\beta_{j, i}$. However, the above equations are impossible to solve. The problem is that we are trying to estimate more parameters than there are observations. While the standard regression model was too restrictive - with the model assumptions not matching the data generating process - this new formulation is not restrictive enough.

The way out of this predicament is to impose structure on the spatial dependence, i.e. to disallow idiosyncratic spatial influences of the predictor variable in each spatial unit on robbery in each spatial unit. For example, we could argue that robbery in the focal hexagon is affected only by the presence of ATMs in the hexagons themselves (the focal hexagon) as well as those that share at least one border with the focal hexagon, and all ATMs in hexagons further away have zero influence on robbery in the focal hexagon. In addition, we assume that spatial neighbors each contribute equally, and that this holds across the entire study area. ${ }^{6}$ We can impose such structure on the spatial dependence by specifying a corresponding spatial weights matrix using 1st-order neighbors of all hexagons covering Washington, D.C., and a "W" (row-standardized) weights matrix (see the Supplementary material for more details). This leads to the spatially-lagged $X$ (SLX) model, where $x_{1}$ refers to the predictor variable $A T M$ :

$$
y_{i}=\beta_{0}+\beta_{1} x_{1, i}+\theta_{1} \sum_{j=1}^{n} W_{i j} x_{1, j}+\epsilon_{i}
$$

The model is the standard OLS regression model, but extended with the term $\theta \sum_{j=1}^{n} W_{i j} x_{1, j}$. The term $\sum_{j=1}^{n} W_{i j} x_{1, j}$ refers to one extra predictor variable that summarizes the ATM presence in all adjacent hexagons, and therefore only one extra parameter, $\theta_{1}$, needs to be estimated as compared to the standard OLS regression model. Of course, this comes at a potential cost: we assume a particular spatial dependency structure, but if we are wrong, $\theta_{1}$ does not capture the spatial process correctly and the parameter estimates $\beta$ and $\theta_{1}$ may be off. Estimating this model results in Table 3 :

\subsection{Taxonomy of spatial regression models}

The SLX model in the example thus far imposes some structure to the spatial dependence at the analyst's discretion, by defining the spatial weights matrix $W$ and calculating one or more

\footnotetext{
${ }^{6}$ One important assumption of the spatial regression models we discuss in this chapter is that the estimated relationships hold across the study area. If they do not, i.e. when some $\beta$ should actually be different in different regions of the study area, this is called spatial heterogeneity. Note that diagnostics for regression residuals react to both spatial dependence and spatial heterogeneity, and therefore they are not very helpful to distinguish the two. There are several ways to deal with heterogeneity. We might first try to include predictors that reflect such local variation (e.g., variables indicating the CBD of Washington D.C., or its wards or neighborhoods). An alternative is Spatial Regimes regression, which allows parameter estimates to be different in different regions. Yet another alternative is to use Geographically Weighted Regression (GWR), in which the relationships between the predictor variables and the outcome variable is allowed to vary more continuously across the study area. Finally, Bayesian Hierarchical ('multilevel') models can be used. These subjects are outside the scope of this chapter, and discussed in several other chapters of this book.
} 
Table 3: SLX Regression Results for robbery

\begin{tabular}{lc}
\hline \hline Liquor licenses & $0.20^{* * *}(0.12,0.29)$ \\
Grocery stores & $2.86^{* *}(1.06,4.66)$ \\
Schools & $4.60^{* * *}(3.51,5.68)$ \\
ATM & $1.21(-1.43,3.85)$ \\
Liquor licenses (lag-1) & $0.05(-0.10,0.19)$ \\
Grocery stores (lag-1) & $2.50(-2.30,7.30)$ \\
Schools (lag-1) & $10.70^{* * *}(8.37,13.04)$ \\
ATM (lag-1) & $-7.10^{*}(-12.58,-1.63)$ \\
Intercept & $-0.32(-1.66,1.01)$ \\
\hline Observations & 274 \\
$\mathrm{R}^{2}$ & 0.63 \\
Adjusted ${ }^{2}$ & 0.62 \\
Residual Std. Error & $6.98(\mathrm{df}=265)$ \\
F Statistic & $56.29^{* * *}(\mathrm{df}=8 ; 265)$ \\
\hline \hline & ${ }^{*} \mathrm{p}<0.05 ;{ }^{* *} \mathrm{p}<0.01 ;{ }^{* * *} \mathrm{p}<0.001$
\end{tabular}

new predictor variables using $W$. The SLX model is just one of many possible ways to model global spatial dependence. As we summarized, there are three main reasons for spatial dependency, and these suggest different regression models: exogenous interaction, endogenous interaction, and interaction among error terms. This section presents a taxonomy of spatial regression models (see Elhorst, 2014, Chapter 1) that aim to capture each of these mechanisms independently or in combination.

The notation for the population regression model described earlier was $y_{i}=\beta_{0}+\beta_{1} x_{1 i}+\beta_{2} x_{2 i}+$ $\cdots+\beta_{k} x_{k i}+\epsilon_{i}$, whereby $i$ indicates the unit of analysis $i=1 . . n$ and there are $k$ predictor variables $x{ }^{7}$ For the next sections, it will be easier to rewrite the regression model in matrix notation, that is, removing the $i$ so it reads: $y=\mathbf{X} \beta+\epsilon$. Here, $y$ refers to a vector (of length $n$ ), $\mathbf{X}$ refers to a matrix of $n$ rows (the $n$ units of analysis) and $k$ columns (the number of predictor variables plus the intercept, which is effectively a vector of 1 's) and $\beta$ is a vector of parameters to be estimated (of length $k$ ), and $\epsilon$ is a vector of disturbance terms. It is exactly the same formula as before, but written more succinctly.

\footnotetext{
${ }^{7}$ To repeat, in practice we use a sample of observations to estimate these population parameters, and such estimated parameters are denoted by $\hat{\beta}$.
} 
Table 4: Taxonomy of regression models

\begin{tabular}{|c|c|c|c|c|c|c|}
\hline $\begin{array}{l}\text { Dep. } \\
\text { var }\end{array}$ & $\begin{array}{c}\text { Non-spatial } \\
\text { Predictor } \\
\text { variables }\end{array}$ & $\begin{array}{c}\text { Spatially-lagged } \\
\text { predictor variables }\end{array}$ & $\begin{array}{l}\text { Spatially-lagged } \\
\text { outcome variable }\end{array}$ & Spatial error & $\begin{array}{l}\text { Random } \\
\text { error }\end{array}$ & Model \\
\hline$y=$ & $\mathbf{X} \beta+$ & & & & $\epsilon$ & (OLS) \\
\hline$y=$ & $\mathbf{X} \beta+$ & $\theta \mathbf{W X}+$ & & & $\epsilon$ & (SLX) \\
\hline$y=$ & $\mathbf{X} \beta+$ & & $\delta \mathbf{W} y+$ & & $\epsilon$ & (SLM) \\
\hline$y=$ & $\mathbf{X} \beta+$ & & & $\lambda \mathbf{W} u+$ & $\epsilon$ & $(\mathrm{SEM})$ \\
\hline$y=$ & $\mathbf{X} \beta+$ & $\theta \mathbf{W X}+$ & $\delta \mathbf{W} y+$ & & $\epsilon$ & $(\mathrm{SDM})$ \\
\hline$y=$ & $\mathbf{X} \beta+$ & & $\delta \mathbf{W} y+$ & $\lambda \mathbf{W} u+$ & $\epsilon$ & $(\mathrm{SAC})$ \\
\hline$y=$ & $\mathbf{X} \beta+$ & $\theta \mathbf{W X}+$ & & $\lambda \mathbf{W} u+$ & $\epsilon$ & (SDEM) \\
\hline$y=$ & $\mathbf{X} \beta+$ & $\theta \mathbf{W X}+$ & $\delta \mathbf{W} y+$ & $\lambda \mathbf{W} u+$ & $\epsilon$ & (GNM) \\
\hline
\end{tabular}

The OLS model is not a spatial regression model but is included for clarity. It is clearly the most restrictive model: it assumes that everything unspecified in this equation is effectively zero. While its simplicity makes it easy to understand and apply, the parameter estimates $\beta$ may be biased when we fail to account for spatial dependence, and thus we may make incorrect inferences.

The spatially-lagged $X$ (SLX) model incorporates the exogenous interaction effect. Because the SLX model offers such a natural way to introduce spatial effects into the standard regression model, this was the model used first in our earlier example. It is simply an OLS model with extra predictor variables, which are spatially-lagged versions of some or all of the predictor variables $x_{1}, x_{2}, \ldots, x_{k}$. The SLX model is not often mentioned in spatial econometrics or spatial statistics books, or only implicitly, likely because it is not challenging to estimate (you can simply use OLS regression). In addition, many theoretical mechanisms in spatial economics - like contagion effects - explicitly relate to spatially-lagged outcome variables. In contrast, in "Crime and Place" studies, with crime as the outcome variable - and perhaps especially when dealing with smaller spatial units - theoretical mechanisms focus more often on nearby environmental features (i.e. predictor variables) and the expected spatial influence of these on crime levels nearby (see, e.g., Bernasco et al., 2012). Although it depends on the theoretical mechanism specified in a study, we recommend not to jump too quickly from a simple OLS model to a spatial lag or spatial error model (see below), but consider spatially-lagged predictor variables first.

The spatial lag model (SLM) incorporates the endogenous interaction effect, where $y$ in each spatial unit depends on the level of $y$ in the units specified in the $W$ matrix, and vice versa. Spatial effects of covariates work indirectly through their influence on crime. The SLM model fits best with theoretical mechanisms like "contagion" or "diffusion" (Cohen \& Tita, 1999; see, e.g., Loftin, 1986; Messner et al., 1999; Papachristos et al., 2015). The idea is similar to the SLX model, but here the weight matrix $W$ is multiplied by the values of $y$. Thus, the model includes the outcome variable on both the left hand side and the right hand side of the equation, which makes this an endogenous model. Because of this endogeneity, this model (and all other models below) can no longer be estimated using OLS. Similar to SLX and all other subsequent models, the spatial weights matrix $W$ needs to be specified by the analyst. The additional parameter to be estimated by SLM is $\delta$.

The first three models assumed that, under different model specifications (respectively, only non-spatial predictor variables in OLS, including spatially-lagged predictor variables in SLX and an endogenous spatial effect in the SLM), the errors $\epsilon$ are independent and identically distributed randomly. The spatial error (SEM) model does not make this assumption, but instead decomposes 
$\epsilon$ into an error component with spatial structure $(u)$ and residual error $(\epsilon)$. Again, the weights matrix needs to be specified by the analyst and the parameter $\lambda$ is estimated. The remaining models incorporate different combinations of the ideas above.

\subsection{Which model should we choose?}

Now that we have presented a variety of different spatial regression specifications, the obvious question that comes to mind is: which model should we choose? Ideally, the choice of model should be guided by theory and knowledge of the data generating process. For example, we argued that there are often hypothesized mechanisms about how nearby environmental features (i.e. predictor variables) affect crime, or that crime can be 'contagious,' suggesting an SLX and SLM model respectively (after first fitting a standard regression model). Theory and empirical evidence can also help guide the choice of how to model the expected spatial influence of these on crime levels nearby, i.e. the choice of spatial neighbors and associated weights.

Realistically speaking, though, regression modeling with observational data will not always follow a prespecified plan of analysis, but the research consists of an iterative process: model diagnostics are used to identify problems, which leads to adjustment of the model, and so on (an excellent reference for the applied researcher is Harrell Jr, 2015). This raises the question of how best to build models. The most common approach to do this is the specific-to-general approach. First, simple models (e.g., OLS) are estimated, leading to respecification in light of violations of the model's assumptions. Then, we turn to more complex models such as the spatial regression models discussed here. In contrast, the general-to-specific approach (e.g., see Hendry \& others, 1995) argues to start with the least restrictive model (GNM) and chip away at this model (effectively restricting certain parameters to be 0) until we end up with a simpler and more parsimonious model. Following the approach of most applied researchers and discussed in most books on regression analysis and spatial regression models, we use the specific-to-general approach in this chapter.

What if there is evidence of spatial dependence after fitting a standard OLS regression model (perhaps after including spatially-lagged predictor variables, and predictor variables that capture spatial heterogeneity)? Lagrange Multiplier test (LM) statistics can be used to suggest whether the data generating process is better captured using a spatial error or a spatial lag model. Anselin (2005, p. 199) provides a decision tree regarding the Lagrange Multiplier test statistics in order to decide upon either a spatial lag or spatial error specification, which we describe here: First, consider the LM-Error and LM-Lag test statistics. If neither rejects the null hypothesis at a prespecified $\alpha$-level, stick to the original model. ${ }^{8}$ If one of these rejects the null hypothesis, estimate the spatial regression model that matches this statistically significant test. Only if both tests reject the null hypothesis, consider the robust forms of the test statistics, RLM-Error and RLMlag. Typically one is statistically significant or magnitudes more significant than the other. Anselin (2005, p. 200) notes that when both are highly statistically significant one should proceed with the model that has the largest value for the test statistic, but it could also hint at other sources of model misspecification.

\footnotetext{
${ }^{8}$ The LM-Error test compares a SEM model to an OLS model, and the null hypothesis states that the $\lambda=0$ in $\lambda \mathbf{W} u$ (see table with taxonomy of models). The LM-Lag test compares a SLM to an OLS model, and the null hypothesis states that $\delta=0$ in $\delta \mathbf{W} y$.
} 
Table 5: Lagrange Multiplier test results

\begin{tabular}{lrrr}
\hline & Statistic & df & p-value \\
\hline LMerr & 53.26 & 1 & 0.00 \\
RLMerr & 1.45 & 1 & 0.23 \\
LMlag & 68.66 & 1 & 0.00 \\
RLMlag & 16.85 & 1 & 0.00 \\
\hline
\end{tabular}

In our example, both LM tests are indeed highly statistically significant with a prespecified $\alpha=.05$. We next consider the robust tests, and here RLM-lag clearly rejects the null hypothesis whereas RLM-error does not. We thus proceed to fit a spatial lag model. Because we also include spatially-lagged predictor variables, this means a Spatial Durbin model is specified, presented in Table $6 .^{9}$

Table 6: SDM regression results for robberies

\begin{tabular}{lc}
\hline \hline Liquor licensee & $0.20^{* * *}(0.13,0.27)$ \\
Grocery stores & $2.74^{* * *}(1.20,4.28)$ \\
Schools & $3.41^{* * *}(2.45,4.36)$ \\
ATM & $1.81(-0.44,4.07)$ \\
Liquor licensee (lag-1) & $-0.12(-0.25,0.01)$ \\
Grocery stores (lag-1) & $1.08(-3.05,5.22)$ \\
Schools (lag-1) & $4.54^{* * *}(2.23,6.85)$ \\
ATM (lag-1) & $-4.22(-8.89,0.46)$ \\
Intercept & $-0.85(-2.00,0.29)$ \\
\hline Observations & 274 \\
Log Likelihood & -887.56 \\
$\sigma^{2}$ & 35.48 \\
Akaike Inf. Crit. & $1,797.12$ \\
Wald Test & $81.29^{* * *}(\mathrm{df}=1)$ \\
LR Test & $58.30^{* * *}(\mathrm{df}=1)$ \\
\hline \hline Note: & \multirow{2}{*}{$\mathrm{p}<0.05 ;{ }^{* *} \mathrm{p}<0.01 ;{ }^{* * *} \mathrm{p}<0.001$} \\
& $\mathrm{Rho:} 0.56$
\end{tabular}

With regard to the choice of models, J. LeSage \& Pace (2009, pp. 157-158) summarize that when the true data generating process is one of SLM, SEM, or SAC, erroneously fitting a SDM model (as well as some of the other models, in some cases) may still produce unbiased parameter estimates. In contrast, when the data generating process does not match the SLM, SEM, or SAC model but these are estimated anyway, in some cases the parameter estimates will be biased. In particular, the SEM model performs badly as compared to the other types of model when the true data generating process is not SEM.

\footnotetext{
${ }^{9}$ Remember that this is an example, in which we assume that these hexagons are the right spatial scale and unit for our process of interest, that the spatial weights matrix $W$ matches the data generating process, and we have a well-specified model. By now the reader should know this is unlikely to be the case, and therefore we emphasize not to put much stock in these results.
} 


\subsection{Interpreting spatial regression models: Impacts}

A final challenge of spatial regression models is the interpretation of the parameters. In OLS regression, $\beta^{\prime} s$ reflect the mean difference in the outcome variable when comparing two groups that differ by one unit on a predictor variable. A more causal interpretation of $\beta$ would be the average "impact" of one unit difference in $x$ on $y$. In spatial regression models with a spatially-lagged outcome variables, such a straightforward interpretation of $\beta$ is no longer possible. A one unit change in a predictor variable cascades throughout the system (J. LeSage \& Pace, 2009): there is a direct impact of $x_{i}$ on $y_{i}$, while $y_{i}$ impacts its neighbor $y_{j}$, which in turn impacts his neighbor $y_{k}$ as well as $y_{i}$. This means $x$ not only directly impacts $y$ in the focal spatial unit, but also indirectly impacts $y$ in other spatial units. All this means that the reader should not interpret the regression results table of models that include a spatially-lagged dependent variable, such as Table 6 .

Instead, we should try to decompose the impact of variables into the average direct impact, the average total impact, and the average indirect impact (J. P. LeSage \& Fischer, 2008; J. LeSage \& Pace, 2009, pp. 33-42). The average direct impact provides a summary measure of the impact of $x_{i}$ on $y_{i}$ averaged over all $i=1, \ldots, n$ spatial units. The average total impact refers to the total cumulative impact of one spatial unit changing $x$ by one unit on $y$ of all other regions, averaged over all spatial units. The average indirect impact is the difference between the total and the direct effect. For the Spatial Durbin Model, this leads to the following impact estimates:

Table 7: Spatial partitioning of direct, indirect and total impacts (SDM)

\begin{tabular}{lrrr}
\hline Variable & Direct Impact & Indirect Impact & Total Impact \\
\hline Liquor licenses & 0.199 & -0.017 & 0.181 \\
Grocery stores & 3.132 & 5.547 & 8.680 \\
Schools & 4.367 & 13.648 & 18.015 \\
ATM & 1.337 & -6.788 & -5.451 \\
\hline
\end{tabular}

\section{Concluding remarks}

In this chapter, we aimed to not just introduce the theory of linear regression analysis but also discuss practical choices and challenges faced by applied researchers. Of course, we had to make a few hard choices of what to describe in detail, quickly mention, or omit entirely. Completely omitted from this chapter are, e.g., transformation of variables, cross-validation, and regularization for models with many predictors. Also omitted in this chapter, but a very useful concept in practice, is the idea to create fake-data simulations using the estimated model to assess model fit (Gelman et al., 2020). These can be very helpful to understand your regression model and its limitations. 
We have tried to be transparent that the models discussed here apply to continuous outcome variables. However, in "Crime and Place" studies, we often want to study outcome variables for which we already know that some of the core assumptions of OLS regression will be violated. Some outcome variables have a restricted range of possible values. Dichotomous $(0 / 1)$ and count $(0,1,2, \ldots)$ variables are two such variable types most commonly found in applied research. For these variables, specific generalized linear models exist. These models use specific functions to link a linear model specification to a non-linear transformation of the outcome variable.

Which model best fits depends on the data generating process that is assumed to have resulted in the outcome variable. Dichotomous outcome variables are most commonly analyzed using a logistic regression model, but other models also exist (e.g, probit, cloglog). Count variables are often analyzed using a Poisson regression model ${ }^{10}$, or the negative binomial regression model if the data are overdispersed. Please know that the interpretation of the regression parameters of these models is different from that of the OLS regression model due to the non-linear relation between the independent variables and the dependent variable. For that reason, it is very important to first thoroughly study how these models work before using them in research (see also Chapter 'Count Regression' in this handbook).

It is particularly helpful to turn to models developed in the field of spatial epidemiology. This field has a similar question to "Crime and Place" studies: they are not so much interested in the count of disease cases in an areal unit, but the count of cases relative to the population at risk (also known as the exposure variable). Regression models are then used to model excess risk, i.e., to understand why some areal units have higher disease incidence than other areal units after accounting for the differences in at-risk population. Translated to "Crime and Place" studies, this means that ideally we would not analyze the number of street robberies per hexagon, but rather the number of street robberies divided by the number of people who are outdoors (the population at risk). ${ }^{11}$ Bivand et al. (2013Ch. 10) provides an introduction to disease mapping and practical applications in R, while Waller \& Gotway (2004Ch. 9) provide an overview of spatial generalized linear models as well as Bayesian models.

What we have not discussed at all in this chapter is that the regression methods discussed are those of the "classical" (a.k.a. "frequentist") variety. Classical statistics is based on estimates and predictions that summarize the information in the data (and only the data). These frequentists are interested in the "long run" expectation of the methods, and this was reflected in our desciption of the parameter estimates and confidence intervals: "Imagine that we would now draw many new random samples and estimate the regression parameters over and over again...." An alternative to classical statistics is "Bayesian" statistics, which uses the data but also incorporates prior information into inferences (i.e. what is a range of parameter estimates that we can reasonably expect before we do the analysis?). The researcher's prior beliefs affect the outcome of the analysis, which can yield valid inferences with weak data, but with additional assumptions. Especially for spatial regression models with very small counts of crime and highly variable at-risk populations across areal units, Bayesian methods often offer advantages over classical methods. A very gentle introduction to Bayesian regression analysis is provided by Gelman et al. (2020) and McElreath (2020), with more advanced material in Gelman et al. (2013). Banerjee et al. (2014) focuses on spatial Bayesian models.

We end with a reflection on science and two ways to approach answering research questions. One

\footnotetext{
${ }^{10}$ The robbery data we analyze in this chapter are actually also an example of a count variable. However, we decided to use the data to explain the linear OLS regression model for didactic reasons.

${ }^{11}$ You can imagine that it is actually quite difficult to accurately measure or estimate the population at risk for street robbery. In contrast, the population at risk for home burglary is the number of residential units per hexagon (relatively easy to obtain).
} 
is the tried-and-true method of hypothesis testing. A researcher derives a testable hypothesis from theory, defines the null hypothesis (which often is, but need not be the hypothesis of zero effect), and tests the hypothesis using empirical data. When the empirical data come from a well-executed randomized controlled-trial, and the hypothesis refers to the expected size of difference between the treatment and control group, then regression - the workhorse of quantitative statistics - can be used to estimate this effect. ${ }^{12}$ Sometimes, it is also possible to use naturally occurring variations that make it appear as if a treatment was randomly assigned. Additional assumptions are needed (and the validity of meeting these assumptions are crucial for causal inference), but such quasiexperiments lend themselves to a relatively straightforward application of regression techniques, such as regression discontinuity and difference-in-difference estimation (for example, see Stock \& Watson, 2020, Chapter 13).

It becomes much more difficult when using observational data and multiple regression models such as we discussed here, because the true data-generating process is often not known. Therefore, we expect that many "Crime and Place" researchers who use regression models in the way we discussed in this chapter will not test a priori specified hypotheses following prespecified analysis plans, but often turn to model building. Plotting the data and simulations from the fitted model as well as diagnostic checks are used to put a model to the test and suggest improvements, after which the model is extended. Rinse and repeat. The take home message is: be complete and transparent in reporting the steps of analysis. While the final model will never be "correct" 13 , the ultimate goal is to understand the phenomena we are interested in. Regression models are an important tool towards this goal.

\section{References}

Anselin, L. (2005). Exploring spatial data with GeoDaTM: A workbook. Center for Spatially Integrated Social Science.

Banerjee, S., Carlin, B. P., \& Gelfand, A. E. (2014). Hierarchical modeling and analysis for spatial data. CRC press.

Bernasco, W., Block, R., \& Ruiter, S. (2012). Go where the money is: modeling street robbers' location choices. Journal of Economic Geography, 13(1), 119-143. https://doi.org/10.1093/jeg/ lbs005

Birch, C. P. D., Oom, S. P., \& Beecham, J. A. (2007). Rectangular and hexagonal grids used for observation, experiment and simulation in ecology [Journal Article]. Ecological Modelling, 206 (3), 347-359. https://doi.org/https://doi.org/10.1016/j.ecolmodel.2007.03.041

Bivand, R. S., Pebesma, E. J., \& Gómez-Rubio, V. (2013). Applied spatial data analysis with r. Second edition. Springer.

Cohen, J., \& Tita, G. (1999). Diffusion in homicide: Exploring a general method for detecting spatial diffusion processes. Journal of Quantitative Criminology, 15(4), 451-493.

Cunningham, S. (2018). Causal inference: The mixtape. Manuscript.

\footnotetext{
${ }^{12}$ Remember the independent $t$-test you were once taught, and immediately forgot? This refers to a test of difference in means between two groups. You can achieve the same result using a regression model with an intercept and a dummy variable $x_{1}$ that denotes to which group a unit of analysis belongs: $y=$ Intercept $+\beta_{1}$ Group!

${ }^{13}$ As statistician George Box put it, "All models are wrong, but some are useful"
} 
Elhorst, J. P. (2014). Spatial econometrics: From cross-sectional data to spatial panels (Vol. 479) [Book]. Springer.

Fotheringham, A. S., \& Wong, D. W. S. (1991). The modifiable areal unit problem in multivariate statistical analysis. Environment and Planning A: Economy and Space, 23(7), 1025-1044.

Gelman, A., Carlin, J. B., Stern, H. S., Dunson, D. B., Vehtari, A., \& Rubin, D. B. (2013). Bayesian data analysis. CRC press.

Gelman, A., Hill, J., \& Vehtari, A. (2020). Regression and other stories. Cambridge University Press.

Harrell Jr, F. E. (2015). Regression modeling strategies: With applications to linear models, logistic and ordinal regression, and survival analysis. Springer.

Hendry, D. F., \& others. (1995). Dynamic econometrics. Oxford University Press on Demand.

Hernán, M. A., \& Robins, J. M. (2020). Causal inference: What if. CRC Boca Raton, FL; https://www.hsph.harvard.edu/miguel-hernan/causal-inference-book/

LeSage, J. P., \& Fischer, M. M. (2008). Spatial growth regressions: Model specification, estimation and interpretation. Spatial Economic Analysis, 3(3), 275-304.

LeSage, J., \& Pace, R. K. (2009). Introduction to spatial econometrics CRC press. Boca Raton, FL.

Loftin, C. (1986). Assaultive violence as a contagious social process. Bulletin of the New York Academy of Medicine, 62(5), 550.

McElreath, R. (2020). Statistical rethinking: A bayesian course with examples in $r$ and stan. CRC press.

Messner, S. F., Anselin, L., Baller, R. D., Hawkins, D. F., Deane, G., \& Tolnay, S. E. (1999). The spatial patterning of county homicide rates: An application of exploratory spatial data analysis. Journal of Quantitative Criminology, 15(4), 423-450.

Papachristos, A. V., Wildeman, C., \& Roberto, E. (2015). Tragic, but not random: The social contagion of nonfatal gunshot injuries. Social Science \& Medicine, 125, 139-150.

R Core Team. (2020). R: A language and environment for statistical computing [Book]. https: //www.R-project.org/

Spiegelhalter, D. (2019). The art of statistics: Learning from data. Penguin UK.

Steiner, P. M., \& Kim, Y. (2016). The mechanics of omitted variable bias: Bias amplification and cancellation of offsetting biases. Journal of Causal Inference, $4(2)$.

Stock, J. H., \& Watson, M. W. (2020). Introduction to econometrics, fourth edition. Pearson Education Ltd.

Tita, G. E., \& Radil, S. M. (2010). Spatial regression models in criminology: Modeling social processes in the spatial weights matrix. In Handbook of quantitative criminology (pp. 101-121). Springer.

Tobler, W. R. (1970). A computer movie simulating urban growth in the detroit region. Economic Geography, 46(sup1), 234-240. 
Waller, L. A., \& Gotway, C. A. (2004). Applied spatial statistics for public health data (Vol. 368). John Wiley \& Sons. 